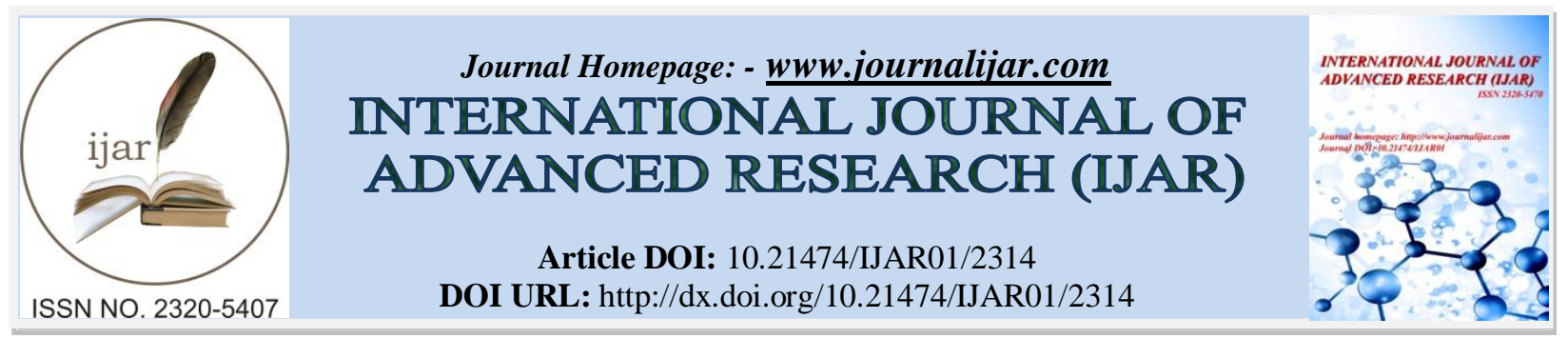

RESEARCH ARTICLE

\title{
AGE- RELATED DIFFERENCES IN REACTION TIME TASK PERFORMANCE IN SCHEDULED CASTE AND SCHEDULED TRIBES CHILDREN OF SONBHADRA DISTRICT.
}

"Tanvi Twara ${ }^{1}$, Sanskriti Upasna ${ }^{2}$, Priyadarshni Tewari ${ }^{3}$, Aruna Agrawal ${ }^{4}$ and G.P Dubey ${ }^{5}$.

1. Junior Research Fellow, PhD Scholar, Dept. of Kriya Sharir, Faculty of Ayurveda, Institute of Medical Sciences, Banaras Hindu University, Varanasi, U.P, India.

2. Junior Research Fellow, PhD Scholar, Dept. of Kriya Sharir, Faculty of Ayurveda, Institute of Medical Sciences, Banaras Hindu University, Varanasi, U.P, India.

3. Assistant Professor, Dept. of Vikriti Vigyan, Faculty of Ayurveda, Institute of Medical Sciences, Banaras Hindu University, Varanasi, U.P, India.

4. Professors, Dept. of Kriya Sharir, Faculty of Ayurveda, Institute of Medical Sciences, Banaras Hindu University, Varanasi, U.P, India.

5. Distinguished Professor, Dept. of Kriya Sharir, Faculty of Ayurveda, Institute of Medical Sciences, Banaras Hindu University, Varanasi, U.P, India.

\section{Manuscript Info}

\section{Manuscript History}

Received: 29 September 2016

Final Accepted: 30 October 2016

Published: November 2016

Key words:-

Reaction time, auditory, visual, processing speed, cognitive.

\section{Abstract}

Background: The present study is an attempt to access the cognitive development of SC and ST children by using non-verbal test of reaction time. Reaction time is defined as the time that passes from the arousal of a sensory organ to a motor reaction. This test help in investigating SC and ST children to see the age related differences in their processing speed. Fast and quick responses show attentive mind and fast processing speed of brain.

Materials and Methods: Present study was conducted on 350 scheduled caste children and 407 scheduled tribe's children with an age group of 4 to 10 years. Study was conducted in villages of Sonbhadra district. For both auditory and visual reaction time three responses from each respondent were recorded in milliseconds (ms). Analysis was done on SPSS by applying independent sample t-test.

Observations \& Result: Mean and standard deviation of VRT and ART of both SC and ST children were compared. While comparing VRT among groups it was found statistically significant in all age groups, but in ART it was found significant only in upper age group i.e 8,9 and 10. Very prolong reaction time was given by both SC and ST children for audio and visual reaction time. Hence from our study we conclude that the cognitive development of both SC and ST is very poor.

Copy Right, IJAR, 2016,. All rights reserved.

\section{Introduction:-}

Reaction time is the time interval between the presentation of the stimulus and appearance of a perfect voluntary response from the subject (1). Concept of reaction time was first described by Abu Rayhan al-Biruni(2). Reaction 
time in human is the measurement of nervous system in recognizing the stimulus. Once the human nervous system gets the stimulus the neurons then convey the message to the brain. The message then travels from brain to the spinal cords which then transfer it to hands and fingers. The motor neurons then guide the hand and fingers, how to react. Reaction time is usually measured in milliseconds. Efficiency of motor action can be predicted by determining the reaction time. Reaction time also helps in making prediction about the stability of attention.

Reaction time is a very important component of our cognitive system. Cognitive development means the ability to think, reason, understand and learn and it also includes memory and recall. Perceptual and sensory skills are part of our cognitive development. In other words cognitive development means the development of five senses, sight, hearing, taste, touch and smell which are further linked to physical development such as development of binocular vision and fine motor skills. The accepted figures for mean simple reaction time for college-age individuals have been about $190 \mathrm{~ms}$ for light stimuli and about $160 \mathrm{~ms}$ for sound stimuli $(\mathbf{3 , 4 , 5 , 6 )}$.

Audio-visual reaction time was investigated in scheduled tribes and scheduled caste children to see the age related differences in their processing speed. Fast reaction time is very important for any task performance and processing requirements. Reaction time is not only an indicator of good health but it can also be used to monitor an individual's condition overtime. Reaction time measures are studied extensively in the laboratory as measures of information-

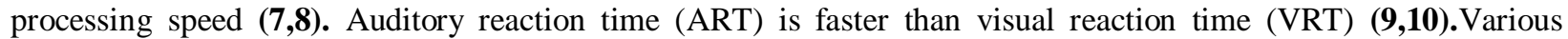
factors affect reaction time like sex, age, physical fitness, type of stimuli, practice, distraction noise, weight, prenatal exposure of alcohol $(\mathbf{1 1 , 1 2 , 1 3 , 1 4 , 1 5 )}$.

\section{Materials and Methods:-}

Three hundred fifty (350) scheduled caste children and four hundred seven (407) scheduled tribe's children in age group 4 to 10 years were selected from various villages of Sonbhadra district. Villages from rural areas were purposively selected with having larger number of SC and ST population. Visits were made to the schools of selected areas and all available subjects lying in our selection criteria were taken. One stage cluster sampling method was implied for collecting data.

Reaction time was measured by using audio-visual reaction time apparatus (Model no.-RTM-608) made by MEDICAID systems. The reaction time range is 0 to $999.9999 \mathrm{sec}$. All the responses given by children were recorded in milliseconds $(\mathrm{msec})$. Both for auditory and visual time reaction three responses were recorded to get more accuracy and mean of these three responses were taken. The readings were taken between $9 \mathrm{am}-4 \mathrm{pm}$ in daylight in silent room. Instrument was kept on table \& subject was made to sit comfortably on chair. Practice was taken from each subject until they have understood and performed the task as required. Subject was asked to press \& immediately release the switch with the thumb of right hand as soon as he saw the glow of yellow light or hearing sound. This gave reaction time in milliseconds on time display of instrument. Each time 3 readings of VRT \& ART were taken and lowest was used as final reading.

Statistical significance of various differences was analyzed by using independent sample t-test. SPSS 16.0 was used for calculating data. We have used levene's test for checking equality of variance and used the t-statistics accordingly. $\mathrm{P}<0.05$ was considered to be statistically significant.

\section{Result:-}

Mean and standard deviation of VRT and ART of both SC and ST children were given in Table 1 and 2. Mean of visual reaction time of both SC and ST were compared in Table 1 by using independent sample t-test and it was found statistically significant in all age groups. From the given table we can also observe that the mean of VRT of SC is lower than the ST in all age group except in age group 4, which means that they were a little faster in giving the responses. 
Table.1:- Mean and standard deviation of visual reaction time of SC and ST children

\begin{tabular}{|c|c|c|c|c|c|c|c|c|}
\hline $\begin{array}{l}\text { Statistical } \\
\text { Significance }\end{array}$ & t-test & $\begin{array}{l}\text { Std. Error } \\
\text { Mean }\end{array}$ & $\begin{array}{l}\text { Std. } \\
\text { Deviation }\end{array}$ & Mean & $\begin{array}{l}\text { No. of } \\
\text { children }\end{array}$ & Caste & \multicolumn{2}{|l|}{ Age } \\
\hline \multirow[t]{2}{*}{ Yes ( $\mathrm{P}$ value 0.034$)$} & 2.149 & 20.859 & 147.492 & 585.82 & 50 & $\begin{array}{l}\text { Scheduled } \\
\text { caste }\end{array}$ & \multirow[t]{2}{*}{ visual } & \multirow[t]{2}{*}{4} \\
\hline & & 17.986 & 134.592 & 526.93 & 56 & $\begin{array}{l}\text { Scheduled } \\
\text { tribes }\end{array}$ & & \\
\hline \multirow[t]{2}{*}{ Yes (P value 0.002$)$} & -3.210 & 8.304 & 62.145 & 503.27 & 56 & $\begin{array}{l}\text { Scheduled } \\
\text { caste }\end{array}$ & \multirow[t]{2}{*}{ visual } & \multirow[t]{2}{*}{5} \\
\hline & & 8.119 & 62.889 & 540.57 & 60 & $\begin{array}{l}\text { Scheduled } \\
\text { tribes }\end{array}$ & & \\
\hline \multirow[t]{2}{*}{ Yes (P value 0.006$)$} & -2.826 & 11.996 & 76.814 & 475.37 & 41 & $\begin{array}{l}\text { Scheduled } \\
\text { caste }\end{array}$ & \multirow[t]{2}{*}{ visual } & \multirow[t]{2}{*}{6} \\
\hline & & 12.389 & 79.330 & 524.10 & 41 & $\begin{array}{l}\text { Scheduled } \\
\text { tribes }\end{array}$ & & \\
\hline \multirow[t]{2}{*}{ Yes (P value 0.000$)$} & -11.917 & 7.013 & 54.326 & 415.68 & 60 & $\begin{array}{l}\text { Scheduled } \\
\text { caste }\end{array}$ & \multirow[t]{2}{*}{ visual } & \multirow[t]{2}{*}{7} \\
\hline & & 6.005 & 51.310 & 525.71 & 73 & $\begin{array}{l}\text { Scheduled } \\
\text { tribes }\end{array}$ & & \\
\hline \multirow[t]{2}{*}{ Yes (P value 0.000$)$} & -11.298 & 6.742 & 45.225 & 400.49 & 45 & $\begin{array}{l}\text { Scheduled } \\
\text { caste }\end{array}$ & \multirow[t]{2}{*}{ visual } & \multirow[t]{2}{*}{8} \\
\hline & & 8.512 & 60.788 & 525.41 & 51 & $\begin{array}{l}\text { Scheduled } \\
\text { tribes }\end{array}$ & & \\
\hline \multirow[t]{2}{*}{ Yes (P value 0.014$)$} & -2.521 & 20.244 & 140.254 & 476.31 & 48 & $\begin{array}{l}\text { Scheduled } \\
\text { caste }\end{array}$ & \multirow[t]{2}{*}{ visual } & \multirow[t]{2}{*}{9} \\
\hline & & 9.157 & 69.137 & 532.32 & 57 & $\begin{array}{l}\text { Scheduled } \\
\text { tribes }\end{array}$ & & \\
\hline \multirow[t]{2}{*}{ Yes (P value 0.011$)$} & -2.602 & 12.980 & 91.782 & 504.74 & 50 & $\begin{array}{l}\text { Scheduled } \\
\text { caste }\end{array}$ & \multirow[t]{2}{*}{ visual } & \multirow[t]{2}{*}{10} \\
\hline & & 7.522 & 62.479 & 543.77 & 69 & $\begin{array}{l}\text { Scheduled } \\
\text { tribes }\end{array}$ & & \\
\hline
\end{tabular}

Whereas in Table 2 mean of audio reaction time of both SC and ST are not statistically significant in all age group. It is significant only in upper age group i.e. 8, 9 and 10. This means that in lower age group the reaction time for audio is same in both SC and ST groups, but significant difference was seen in the upper age between the groups. SC children response on audio reaction time was quick and fast in comparison to ST children in age group 4, 5, 6, 9 and 10 only.

Table. 2:- Mean and standard deviation of audio reaction time of SC and ST children.

\begin{tabular}{|c|c|c|c|c|c|c|c|c|}
\hline $\begin{array}{l}\text { Statistical } \\
\text { Significance }\end{array}$ & t-test & $\begin{array}{l}\text { Std. } \\
\text { Error } \\
\text { Mean }\end{array}$ & $\begin{array}{l}\text { Std. } \\
\text { Deviation }\end{array}$ & Mean & $\begin{array}{l}\text { No. of } \\
\text { children }\end{array}$ & Caste & \multicolumn{2}{|l|}{ Age } \\
\hline \multirow[t]{2}{*}{ No (P value 0.657$)$} & -0.446 & 21.400 & 151.322 & 716.56 & 50 & $\begin{array}{l}\text { Scheduled } \\
\text { caste }\end{array}$ & \multirow[t]{2}{*}{ Audio } & \multirow[t]{2}{*}{4} \\
\hline & & 18.821 & 140.843 & 729.27 & 56 & $\begin{array}{l}\text { Scheduled } \\
\text { tribes }\end{array}$ & & \\
\hline \multirow[t]{2}{*}{ No (P value 0.529$)$} & -0.631 & 16.227 & 121.435 & 695.57 & 56 & $\begin{array}{l}\text { Scheduled } \\
\text { caste }\end{array}$ & \multirow[t]{2}{*}{ Audio } & \multirow[t]{2}{*}{5} \\
\hline & & 21.460 & 166.229 & 712.55 & 60 & $\begin{array}{l}\text { Scheduled } \\
\text { tribes }\end{array}$ & & \\
\hline \multirow[t]{2}{*}{ No (P value 0.702$)$} & -0.384 & 19.631 & 125.699 & 697.66 & 41 & $\begin{array}{l}\text { Scheduled } \\
\text { caste }\end{array}$ & \multirow[t]{2}{*}{ Audio } & \multirow[t]{2}{*}{6} \\
\hline & & 20.688 & 132.465 & 708.61 & 41 & $\begin{array}{l}\text { Scheduled } \\
\text { tribes }\end{array}$ & & \\
\hline No (P value 0.705$)$ & 0.379 & 16.440 & 127.347 & 684.42 & 60 & $\begin{array}{l}\text { Scheduled } \\
\text { caste }\end{array}$ & Audio & 7 \\
\hline
\end{tabular}




\begin{tabular}{|c|c|c|c|c|c|c|c|c|}
\hline & & 13.171 & 112.534 & 676.53 & 73 & $\begin{array}{l}\text { Scheduled } \\
\text { tribes }\end{array}$ & & \\
\hline Yes (P value 0.000$)$ & 3.652 & 19.773 & 132.641 & 702.98 & 45 & $\begin{array}{l}\text { Scheduled } \\
\text { caste }\end{array}$ & \multirow[t]{2}{*}{ Audio } & \multirow[t]{2}{*}{8} \\
\hline & & 15.023 & 107.289 & 612.29 & 51 & $\begin{array}{l}\text { Scheduled } \\
\text { tribes }\end{array}$ & & \\
\hline Yes (P value 0.000$)$ & 3.595 & 17.605 & 121.969 & 511.50 & 48 & $\begin{array}{l}\text { Scheduled } \\
\text { caste }\end{array}$ & \multirow[t]{2}{*}{ Audio } & \multirow[t]{2}{*}{9} \\
\hline & & 14.530 & 109.700 & 592.82 & 57 & $\begin{array}{l}\text { Scheduled } \\
\text { tribes }\end{array}$ & & \\
\hline Yes (P value 0.000$)$ & 3.305 & 14.883 & 105.237 & 532.36 & 50 & $\begin{array}{l}\text { Scheduled } \\
\text { caste }\end{array}$ & \multirow[t]{2}{*}{ Audio } & \multirow[t]{2}{*}{10} \\
\hline & & 13.919 & 115.619 & 600.72 & 69 & $\begin{array}{l}\text { Scheduled } \\
\text { tribes }\end{array}$ & & \\
\hline
\end{tabular}

In Table 3 and 4 comparisons between SC and ST for both VRT and ART was done in female and male children respectively. In table 3 a significant difference was seen between SC and ST females in age group 4, 5, 7 and 8 while comparing VRT, whereas in ART it is significant only in age group 5 and 9. Form the given table we can see that the same age group of female with different groups were performed better and fast in the visual reaction time than from audio reaction time. This is same with the male children they also performed fast and quick in VRT than in ART in almost all age groups.

In table 4 a significant difference was seen between SC and ST male children in age group 6, 7, 8 and 9 while comparing VRT, whereas in ART it was significant in all age group except in age 6. But the mean of VRT and ART of SC and ST children was very high in comparison to accepted figures for mean simple reaction time. This result revealed prolonged reaction time in both SC and ST children for VRT and ART.

Table 3:- Age related comparison between the female children of SC and ST groups in both audio and visual reaction time.

\begin{tabular}{|c|c|c|c|c|c|c|c|c|}
\hline$P$ value & t-test & $\begin{array}{l}\text { Std. Error } \\
\text { Mean }\end{array}$ & $\begin{array}{l}\text { Std. } \\
\text { Deviation }\end{array}$ & Mean & $\begin{array}{l}\text { No. of } \\
\text { children }\end{array}$ & Caste & \multicolumn{2}{|l|}{ Age } \\
\hline $\begin{array}{l}\text { Yes (P value } \\
0.023)\end{array}$ & 2.334 & 31.838 & 162.343 & 622.23 & 26 & $\begin{array}{l}\text { Scheduled } \\
\text { caste }\end{array}$ & \multirow[t]{2}{*}{ visual } & \multirow[t]{4}{*}{4} \\
\hline & & 29.336 & 157.977 & 521.34 & 29 & $\begin{array}{l}\text { Scheduled } \\
\text { tribes }\end{array}$ & & \\
\hline $\begin{array}{l}\text { No (P value } \\
0.099)\end{array}$ & 1.677 & 25.954 & 132.341 & 792.04 & 26 & $\begin{array}{l}\text { Scheduled } \\
\text { caste }\end{array}$ & \multirow[t]{2}{*}{ audio } & \\
\hline & & 26.428 & 142.318 & 729.66 & 29 & $\begin{array}{l}\text { Scheduled } \\
\text { tribes }\end{array}$ & & \\
\hline $\begin{array}{ll}\text { Yes (P value } \\
0.000)\end{array}$ & -4.861 & 10.776 & 58.031 & 471.21 & 29 & $\begin{array}{l}\text { Scheduled } \\
\text { caste }\end{array}$ & \multirow[t]{2}{*}{ visual } & \multirow[t]{4}{*}{5} \\
\hline & & 11.513 & 63.058 & 547.97 & 30 & $\begin{array}{l}\text { Scheduled } \\
\text { tribes }\end{array}$ & & \\
\hline $\begin{array}{ll}\text { Yes } & (\mathrm{P} \text { value } \\
0.000) & \end{array}$ & -5.276 & 14.310 & 77.063 & 642.69 & 29 & $\begin{array}{l}\text { Scheduled } \\
\text { caste }\end{array}$ & \multirow[t]{2}{*}{ audio } & \\
\hline & & 27.112 & 148.497 & 804.43 & 30 & $\begin{array}{l}\text { Scheduled } \\
\text { tribes }\end{array}$ & & \\
\hline $\begin{array}{l}\text { No (P value } \\
0.318)\end{array}$ & -1.011 & 21.046 & 96.444 & 494.19 & 21 & $\begin{array}{l}\text { Scheduled } \\
\text { caste }\end{array}$ & \multirow[t]{2}{*}{ visual } & \multirow[t]{4}{*}{6} \\
\hline & & 12.195 & 54.536 & 519.10 & 20 & $\begin{array}{l}\text { Scheduled } \\
\text { tribes }\end{array}$ & & \\
\hline $\begin{array}{l}\text { No (P value } \\
0.893)\end{array}$ & 0.135 & 23.355 & 107.028 & 720.62 & 21 & $\begin{array}{l}\text { Scheduled } \\
\text { caste }\end{array}$ & \multirow[t]{2}{*}{ audio } & \\
\hline & & 29.576 & 132.269 & 715.55 & 20 & $\begin{array}{l}\text { Scheduled } \\
\text { tribes }\end{array}$ & & \\
\hline Yes $\quad(\mathrm{P} \quad$ value & -8.418 & 10.433 & 57.143 & 437.33 & 30 & Scheduled & visual & 7 \\
\hline
\end{tabular}




\begin{tabular}{|c|c|c|c|c|c|c|c|c|}
\hline 0.000$)$ & & & & & & caste & & \\
\hline & & 6.811 & 42.534 & 538.31 & 39 & $\begin{array}{l}\text { Scheduled } \\
\text { tribes }\end{array}$ & & \\
\hline $\begin{array}{ll}\text { No }(\mathrm{P} & \text { value } \\
0.099) & \end{array}$ & -1.674 & 17.793 & 97.457 & 638.90 & 30 & $\begin{array}{l}\text { Scheduled } \\
\text { caste }\end{array}$ & audio & \\
\hline & & 19.387 & 121.069 & 684.21 & 39 & $\begin{array}{l}\text { Scheduled } \\
\text { tribes }\end{array}$ & & \\
\hline $\begin{array}{l}\text { Yes (P value } \\
0.000)\end{array}$ & -7.674 & 10.002 & 47.966 & 413.65 & 23 & $\begin{array}{l}\text { Scheduled } \\
\text { caste }\end{array}$ & visual & 8 \\
\hline & & 10.949 & 58.961 & 530.21 & 29 & $\begin{array}{l}\text { Scheduled } \\
\text { tribes }\end{array}$ & & \\
\hline $\begin{array}{ll}\text { No }(P & \text { value } \\
0.315) & \end{array}$ & 1.015 & 25.652 & 123.024 & 667.74 & 23 & $\begin{array}{l}\text { Scheduled } \\
\text { caste }\end{array}$ & audio & \\
\hline & & 21.796 & 117.375 & 633.76 & 29 & $\begin{array}{l}\text { Scheduled } \\
\text { tribes }\end{array}$ & & \\
\hline $\begin{array}{l}\text { No (P value } \\
0.349)\end{array}$ & 0.947 & 33.551 & 153.750 & 580.95 & 21 & $\begin{array}{l}\text { Scheduled } \\
\text { caste }\end{array}$ & visual & 9 \\
\hline & & 15.234 & 74.633 & 547.50 & 24 & $\begin{array}{l}\text { Scheduled } \\
\text { tribes }\end{array}$ & & \\
\hline $\begin{array}{l}\text { No (P value } \\
0.106)\end{array}$ & -1.660 & 29.864 & 136.855 & 523.71 & 21 & $\begin{array}{l}\text { Scheduled } \\
\text { caste }\end{array}$ & audio & \\
\hline & & 17.981 & 88.090 & 581.58 & 24 & $\begin{array}{l}\text { Scheduled } \\
\text { tribes }\end{array}$ & & \\
\hline $\begin{array}{l}\text { No (P value } \\
0.069)\end{array}$ & -1.875 & 20.40 & 95.71 & 496.32 & 22 & $\begin{array}{l}\text { Scheduled } \\
\text { caste }\end{array}$ & visual & 10 \\
\hline & & 12.40 & 73.40 & 541.09 & 35 & $\begin{array}{l}\text { Scheduled } \\
\text { tribes }\end{array}$ & & \\
\hline $\begin{array}{l}\text { No (P value } \\
0.137)\end{array}$ & -1.511 & 19.79 & 92.843 & 567.14 & 22 & $\begin{array}{l}\text { Scheduled } \\
\text { caste }\end{array}$ & audio & \\
\hline & & 20.996 & 124.214 & 613.69 & 35 & $\begin{array}{l}\text { Scheduled } \\
\text { tribes }\end{array}$ & & \\
\hline$P$ value & t-test & $\begin{array}{l}\text { Std. Error } \\
\text { Mean }\end{array}$ & $\begin{array}{l}\text { Std. } \\
\text { Deviation }\end{array}$ & Mean & $\begin{array}{l}\begin{array}{l}\text { No. of } \\
\text { children }\end{array} \\
\end{array}$ & Caste & Age & \\
\hline $\begin{array}{l}\text { No (P value } \\
0.675)\end{array}$ & 0.42 & 24.644 & 120.730 & 546.38 & 24 & $\begin{array}{l}\text { Scheduled } \\
\text { caste }\end{array}$ & visual & 4 \\
\hline & & 20.522 & 106.637 & 532.93 & 27 & $\begin{array}{l}\text { Scheduled } \\
\text { tribes }\end{array}$ & & \\
\hline $\begin{array}{lll}\text { Yes } & (\mathrm{P} & \text { value } \\
0.01) & & \end{array}$ & -2.47 & 26.084 & 127.786 & 634.79 & 24 & $\begin{array}{l}\text { Scheduled } \\
\text { caste }\end{array}$ & audio & \\
\hline & & 27.318 & 141.951 & 728.85 & 27 & $\begin{array}{l}\text { Scheduled } \\
\text { tribes }\end{array}$ & & \\
\hline $\begin{array}{l}\text { No }(\mathrm{P} \text { value } \\
0.76)\end{array}$ & 0.307 & 8.954 & 46.529 & 537.70 & 27 & $\begin{array}{l}\text { Scheduled } \\
\text { caste }\end{array}$ & visual & 5 \\
\hline & & 11.484 & 62.903 & 533.17 & 30 & $\begin{array}{l}\text { Scheduled } \\
\text { tribes }\end{array}$ & & \\
\hline $\begin{array}{lll}\text { Yes } & (\mathrm{P} \quad \text { value } \\
0.00) & & \\
\end{array}$ & 3.75 & 26.055 & 135.387 & 752.37 & 27 & $\begin{array}{l}\text { Scheduled } \\
\text { caste }\end{array}$ & audio & \\
\hline & & 23.592 & 129.221 & 620.67 & 30 & $\begin{array}{l}\text { Scheduled } \\
\text { tribes }\end{array}$ & & \\
\hline $\begin{array}{lll}\text { Yes } & (\mathrm{P} \quad \text { value } \\
0.00) & & \end{array}$ & -3.06 & 9.560 & 42.754 & 455.60 & 20 & $\begin{array}{l}\text { Scheduled } \\
\text { caste }\end{array}$ & visual & 6 \\
\hline & & 21.506 & 98.551 & 528.86 & 21 & $\begin{array}{l}\text { Scheduled } \\
\text { tribes }\end{array}$ & & \\
\hline (P value & -0.65 & 31.632 & 141.462 & 673.55 & 20 & Scheduled & audio & \\
\hline
\end{tabular}




\begin{tabular}{|c|c|c|c|c|c|c|c|c|}
\hline 0.51$)$ & & & & & & caste & & \\
\hline & & 29.584 & 135.572 & 702.00 & 21 & $\begin{array}{l}\text { Scheduled } \\
\text { tribes }\end{array}$ & & \\
\hline $\begin{array}{lll}\text { Yes } & (\mathrm{P} \quad \text { value } \\
0.00) & \end{array}$ & -9.24 & 7.680 & 42.064 & 394.03 & 30 & $\begin{array}{l}\text { Scheduled } \\
\text { caste }\end{array}$ & \multirow[t]{2}{*}{ visual } & \multirow[t]{4}{*}{7} \\
\hline & & 9.789 & 57.081 & 511.26 & 34 & $\begin{array}{l}\text { Scheduled } \\
\text { tribes }\end{array}$ & & \\
\hline $\begin{array}{lll}\text { Yes } & (\mathrm{P} & \text { value } \\
0.04) & & \\
\end{array}$ & 2.01 & 25.304 & 138.598 & 729.93 & 30 & $\begin{array}{l}\text { Scheduled } \\
\text { caste }\end{array}$ & \multirow[t]{2}{*}{ audio } & \\
\hline & & 17.659 & 102.969 & 667.74 & 34 & $\begin{array}{l}\text { Scheduled } \\
\text { tribes }\end{array}$ & & \\
\hline $\begin{array}{lll}\text { Yes } & (\mathrm{P} \quad \text { value } \\
0.00) & & \\
\end{array}$ & -8.31 & 8.221 & 38.560 & 386.73 & 22 & $\begin{array}{l}\text { Scheduled } \\
\text { caste }\end{array}$ & \multirow[t]{2}{*}{ visual } & \multirow[t]{4}{*}{8} \\
\hline & & 13.633 & 63.946 & 519.09 & 22 & $\begin{array}{l}\text { Scheduled } \\
\text { tribes }\end{array}$ & & \\
\hline $\begin{array}{lll}\text { Yes } & (\mathrm{P} \quad \text { value } \\
0.00) & & \\
\end{array}$ & 4.55 & 28.782 & 135.002 & 739.82 & 22 & $\begin{array}{l}\text { Scheduled } \\
\text { caste }\end{array}$ & \multirow[t]{2}{*}{ audio } & \\
\hline & & 18.541 & 86.964 & 584.00 & 22 & $\begin{array}{l}\text { Scheduled } \\
\text { tribes }\end{array}$ & & \\
\hline $\begin{array}{lll}\text { Yes } & (\mathrm{P} \quad \text { value } \\
0.00) & & \\
\end{array}$ & -8.91 & 7.825 & 40.659 & 394.93 & 27 & $\begin{array}{l}\text { Scheduled } \\
\text { caste }\end{array}$ & \multirow[t]{2}{*}{ visual } & \multirow[t]{4}{*}{9} \\
\hline & & 11.096 & 63.740 & 521.27 & 33 & $\begin{array}{l}\text { Scheduled } \\
\text { tribes }\end{array}$ & & \\
\hline $\begin{array}{lll}\text { Yes } & (\mathrm{P} & \text { value } \\
0.00) & & \\
\end{array}$ & -3.23 & 21.319 & 110.774 & 502.00 & 27 & $\begin{array}{l}\text { Scheduled } \\
\text { caste }\end{array}$ & \multirow[t]{2}{*}{ audio } & \\
\hline & & 21.544 & 123.767 & 601.00 & 33 & $\begin{array}{l}\text { Scheduled } \\
\text { tribes }\end{array}$ & & \\
\hline $\begin{array}{l}\text { No }(\mathrm{P} \text { value } \\
0.07)\end{array}$ & -1.85 & 16.966 & 89.775 & 511.36 & 28 & $\begin{array}{l}\text { Scheduled } \\
\text { caste }\end{array}$ & \multirow[t]{2}{*}{ visual } & \multirow[t]{4}{*}{10} \\
\hline & & 8.534 & 49.761 & 546.53 & 34 & $\begin{array}{l}\text { Scheduled } \\
\text { tribes }\end{array}$ & & \\
\hline $\begin{array}{lll}\text { Yes } & (\mathrm{P} \quad \text { value } \\
0.00) & & \\
\end{array}$ & -3.01 & 20.384 & 107.865 & 505.04 & 28 & $\begin{array}{l}\text { Scheduled } \\
\text { caste }\end{array}$ & \multirow[t]{2}{*}{ audio } & \\
\hline & & 18.220 & 106.241 & 587.38 & 34 & $\begin{array}{l}\text { Scheduled } \\
\text { tribes }\end{array}$ & & \\
\hline
\end{tabular}

Table 4:- Age related comparison between the male children of SC and ST groups in both audio and visual reaction time.

\section{Discussion:-}

In our study, the reaction time was very longer for the responses to the auditory and visual stimuli from both group's children. The cause of this was their less attentive minds and slow information processing speeds. Most likely it was because of the environment in which they were growing. Various environmental factors have been shown to influence the development of cognitive, language and behavioral in infant and children like maternal IQ, education level, and socioeconomic status (SES) of the parents, quality of the home environment (16), maternal knowledge of the child development and maternal coping (17). All of these environmental factors were lacking in our study group. In our study we observed that their only interest in going school was because of the mid-day meal provided by the government. Children were mostly available in the early hours of school and after eating meal they mostly escape the school. On the other hand their education lacks various exercises and activities which are very important for cognitive development. Our study data reveals that in VRT SC children response were little faster than the ST children, and hence their comparison were found statistically significant in all age groups. Where as in ART SC and ST comparison was statistically significant only in the upper age groups ( 8,9 and 10 years). In ART also SC children performance was little better than ST children but not in all age groups. Most disappointing thing which we observe in our study was that with an increase in the age there was not much difference in their responses.

Researchers use reaction time to examine recognition of their subjects. Kircher et al. (2001) used a reaction time experiment to examine recognition in his samples. Hence their slow performance in both ART and VRT shows a 
very low recognition power in them. They couldn't recognize three different sounds for three different buttons. Recognizing three different buttons for three different colors was easy but not for all. McEvoy, Pellouchoud, Smith and Gevins (2001) conduct reaction time experiment to study memory whereas Stuss et al. (1999) use it for exploring attention. Hence slow responses in both ART and VRT means low memory and attention in children. Both SC and ST children had taken very longer time in giving responses to the stimulus.

Another most important reason for their slow reaction time is the lack of good anticipatory skill. Children living in the rural areas of the Sonbhadra district weren't very comfortable with these instruments. Despite of our best efforts in making them comfortable with us they were not. They highly lack confidence. Lack of proper nutrition is also another most important cause for this. Children belonging to poor socio cultural environment suffer highly from nutritional deficiencies and infections which adversely affect their intellectual and behavioral development (21, 22). These children because of the overcrowded homes with poor sanitation and water supply, low income, limited parent's education and intelligence and a little knowledge of child development and importance of play may also suffer from depression.

\section{Conclusion:-}

From our study we conclude that the cognitive development in SC and ST children is very poor. Children were taking very long time in giving responses to the stimulus. Their performance in both VRT and ART was very bad. There wasn't much difference in 4 years and 10 year children response and also not much difference between the genders in both groups. Low anticipatory skills, less attentive minds and slow information processing speeds are the main reason for their poor performance. Various other factors are all there which affect their performance like physical unfit, lack of practice, weight and poor nutritional status. These children aren't very social in nature they rarely get the chance to interact with urban people and their lifestyle. Hence they weren't able to mix up with us properly in spite of our all efforts. This may be one of the reasons for their slow performance. Scheduled caste and scheduled tribes wherever they are living they are facing a lots of problem. In our society almost all activities like economical, political, educational and socio-cultural revolve around the notions of caste. This structural form and relationships has come to some change in the urban setting but not in rural. Hence our government should require paying attention on this group. Proper education facilities with various exercise and activities which help the children in improving their scholastics performance were provided to them.

\section{Acknowledgments:-}

This study was carried out under an on-going DST project "Effect of malnutrition on cognitive development of tribal children of eastern Uttar Pradesh with especial reference to psychosomatic constitution". The authors acknowledge Dr. T.B Singh and Dr. Grish Singh from the department of Bio-statistic for helping in data calculation on SPSS.

\section{References:-}

1. Teichner WH. The recent studies on the simple reaction time. Psychol Bull 1954; 51: 128-49.

2. Callan JR, Holloway FA. Effects of distraction upon reaction time performance in brain-damaged and alcoholic patients. Neuropsychologia 1972; 10: 363-370.

3. Galton, F. 1899. On instruments for (1) testing perception of differences of tint and for (2) determining reaction time. Journal of the Anthropological Institute 19: 27-29.

4. Fieandt, K. von, A. Huhtala, P. Kullberg, and K. Saarl. 1956. Personal tempo and phenomenal time at different age levels. Reports from the Psychological Institute, No. 2, University of Helsinki.

5. Welford, A. T. 1980. Choice reaction time: Basic concepts. In A. T. Welford (Ed.), Reaction Times. Academic Press, New York, pp. 73-128.

6. Brebner, J. T. and A. T. Welford. 1980. Introduction: an historical background sketch. In A. T. Welford (Ed.), Reaction Times. Academic Press, New York, pp. 1-23.

7. T. Gabbett, M. Rubinoff, L. Thorburn, and D. Farrow, "Testing and training anticipation skills in softball fielders," International Journal of Sports Science \& Coaching, vol. 2, no. 1, pp. 15-24, 2007.

8. M. Audiffren, P. D. Tomporowski, and J. Zagrodnik, "Acute aerobic exercise and information processing: Energizing motor processes during a choice reaction time task," Acta Psychologica, vol. 129, pp. 410-419, 2008.

9. Annie WY, Alan HS, Chan HS. Finger Response Times to Visual, Auditory and Tactile Modality Stimuli. Preceedings of the International Multi conference of Engineers and Computer Scientists 2012; IMECS 2012, March 14-16, 2012, Hong Kong. 
10. Shelton J, Kumar GP. Comparison between Auditory and Visual Simple Reaction Times. Neuroscience and Medicine 2010; 1: 30-32.

11. Knott VJ. Reaction time, noise distraction and autonomic responsivity in smokers and non-smokers. Percept Mot Skills 1980; 50: 1271-1280.

12. Gardner RM, Maes JL, Sandoval Y, Dalsing S. Effects of distraction and task complexity on reaction time in obese persons. Percept Mot Skills 1985; 61: 855-861.

13. Streissguth AP, Barr HM, Sampson PD, Parrish-Johnson JC, Kirchner GL, Martin DC. Attention, distraction and reaction time at age 7 years and prenatal alcohol exposure. Neuro behav Toxicol Teratol 1986; 8: 717- 725 .

14. Ohman A, Nordby H, d'Elia G. Orienting and schizophrenia: stimulus significance, attention, and distraction in a signaled reaction time task. J Abnorm Psychol 1986; 95:326-334.

15. Wallace RM, Fehr FS. Heart rate, skin resistance, and reaction time of mongoloid and normal children under baseline and distraction conditions. Psychophysiology1970; 6: 722-731.

16. Bacharach, V. R., \& Baumeister, A. A. (1998). Direct and indirect effects of maternal intelligence, maternal age, income, and home environment on intelligence of preterm low-birth-weight children. Journal of Applied Developmental Psychology, 19(3), 361-375.

17. Veddovi, M., Gibson, F., Kenny, D. T., Bowen, J., \& Starte, D. (2004). Preterm behavior, maternal adjustment, and competencies in the newborn period: What influence do they have at 12 months postnatal age? Infant Mental Health Journal, 25(6), 580-599.

18. Kircher, T.T.J., Senior, C., Phillips, M.L., Rabe-Hesketh, S., Benson, P.J., Bullmore, E.T., Brammer, M., Simmons, A., Bartels, M. and David, A.S. (2001). Recognizing one's own face. Cognition, 78 (1), B1-B15.

19. McEvoy, L.K., Pellouchoud, E., Smith, M.E. and Gevins, A. (2001). Neurophysiological signals of working memory in normal aging. Cognitive Brain Research, 11 (3), 363-376.

20. Stuss, D.T., Toth, J.P., Franchi, D., Alexander, M.P., Tipper, S. and Craik, F.I.M. (1999). Dissociation of attentional processes in patients with focal frontal and posterior lesions. Neuropsychologia, 37 (9), 1005-1027.

21. Grantham-McGregor S. Social background in childhood malnutrition. In: Brozeck J, Schurch B, eds. Malnutrition and behaviour: critical assessments and key issues.Lausanne, Switzerland: Nestlé Foundation, 1984:358-74.

22. Richardson S. The background histories of schoolchildren severely malnourished in infancy. Adv Pediatr 1974;21:167-95. 